PTU-75 A CLINICAL AUDIT OF SEVERITY ASSESSMENT OF ACUTE PANCREATITIS USING SCORING SYSTEMS

Izza Abid*, Nicholas Cooper, Maitham Alwhouhayb, Pawan Mathur. Royal Free London Nhs Foundation Trust, Barnet, London, UK

\subsection{6/gutjnl-2021-BSG.277}

Introduction Acute Pancreatitis has a wide spectrum of illness, ranging from mild disease to severe disease that may require cardiorespiratory support in ITU. Severity should be predicted using scoring systems such as Glasgow Imrie (GI) and Apache II on admission so as to treat possibly severe cases more aggressively and prevent mortality.

Methods We performed a closed loop audit on the practice of scoring Acute Pancreatitis patients on admission using Glasgow Imrie (GI) and Apache II systems. The data of 97 patients was collected who had been admitted to the General Surgery department at Barnet Hospital with Acute Pancreatitis, irrespective of aetiology. We retrospectively assessed them with GI and Apache II, and correlated the score with the outcome of hospital stay in terms of recovery, ITU admission and mortality. The data was analysed and presented at our General Surgery Audit meeting. Based on the analysis, we implemented in our management, protocol for all patients admitted with Acute Pancreatitis to be scored for severity using GI and Apache II systems on admission. We then collected prospective data to complete the audit cycle.

Results The analysis of initial data revealed that only $65 \%$ of the 97 patients had been scored on admission using the Glasgow Imrie system. Apache II had not been used to score any patient. After retrospective scoring, 93 out of 97 patients were found to have Mild to Moderate Pancreatitis by GI while 78 were assessed as Mild to Moderate by Apache II. $9.6 \%$ of GI Mild cases required ITU admission and 5.3\% ended in mortality. Compared to this, only $6.4 \%$ of Apache Mild patients had been admitted to ITU and only 3.8\% died during hospital stay. Post- implementation of scoring protocol, we have recorded data every 2 weeks showing an improvement in number of patients scored. In the 1st two weeks, $55 \%$ of patients were scored using GI on admission and $33 \%$ were scored using Apache II. In the next two weeks, the respective percentages of scored patients increased to $77.8 \%$ and $33.3 \%$. For all these cases, there was 0\% ITU admission or mortality for patients who were scored and managed accordingly.

Conclusions It is vital to predict severity of disease in patients admitted with Acute Pancreatitis. Recognizing severe illness leads to more aggressive management and prevents ITU admission and mortality.

\section{PTU-76 PREVALENCE AND FOLLOW-UP OF MUCINOUS PANCREATIC CYSTIC LESIONS IN A COHORT OF INPATIENTS}

Almoiez Eltahir*, Velauthan Rudralingam, Venkata Lekharaju, Javaid Iqbal, Sajjad Mahmood. Manchester University NHS Foundation Trust, Manchester, UK

\subsection{6/gutjnl-2021-BSG.278}

Introduction There has been an increasing interest in pancreatic cystic lesions over the past couple of decades due to malignant potential of certain types of pancreatic cysts particularly intraductal papillary mucinous neoplasms (IPMNs) and mucinous cystic neoplasms (MCNs) of the pancreas. A previous study showed prevalence of cystic lesions was 9.3\% in a cohort of patients attending for Magnetic resonance imaging [1]. A prospective population-based Study using magnetic resonance cholangiopancreaticography (MRCP) showed a prevalence of $49.1 \%$ for pancreatic cysts with a criteria of diameter $\geq 2 \mathrm{~mm}$ and a 5 -year follow-up revealed an incidence of $2.6 \%$ per year in general population [2].

We present our data for patients who were identified to have pancreatic cystic lesions during inpatient episodes and follow-up subsequently for suspected mucinous lesions.

Methodology Data search was performed using the ICD-10 codes D13.6 (Benign neoplasm of pancreas), K86.2 (Cyst of pancreas) and K86.3 (Pseudocyst of pancreas).

Demographics, diagnosis and follow up data of patients with pancreatic cystic lesions identified between 2011 and 2020 were analysed. Data regarding diagnostics such as Endoscopic ultrasound (EUS) and fine needle aspiration (FNA) and MDT discussions were recorded. Histopathological findings were also recorded for those who were referred for surgery.

Results Over a 10-year period a total of 162 inpatients were identified as having pancreatic cystic lesions. The age range was 20-91 years with an average of 66 years, 63\% were over the age of 65 years and $42 \%$ were males. The median followup duration was 4 years.

$42 \%$ patients $(n=68)$ had pseudocysts. 33\% $(n=55)$ were found to have IPMNs, of these 49 patients had side-branch IPMNs, 4 patients had main-duct IPMNs and 2 patients had mixed IPMN. MCN and serous cystadenomas were identified in $4 \%$ each. Other lesions include simple pancreatic cysts (20 patients), pancreatic neuroendocrine tumors (NETs, 3 patients), pancreatic cancers (4 patients) and indeterminate lesions (one patient) accounted for $17 \%$ of the total number of patients.

7 patients $(4.3 \%)$ were referred for surgery, 4 had invasive adenocarcinoma and one patient had high grade dysplasia.

Conclusion Prevalence of mucinous neoplastic lesions in this cohort of patients with Pancreatic Cystic Lesions was 38\%. $4.3 \%$ of patients who needed surgery were main-duct IPMN (3patients) and mucinous cystic neoplasms (4 patients). The most common histological type was invasive adenocarcinomas. Interestingly, no patients with side-branch IPMN needed surgical resection in this cohort suggesting a relatively low risk of progression to malignancy. This data may help planning for follow up services for this cohort of patients.

\section{REFERENCES}

1. de Oliveira PB, Puchnick A, Szejnfeld J, et al. Prevalence of incidental pancreatic cysts on 3 tesla magnetic resonance. PLoS One 2015;10:e0121317

2. Kromrey $M-L$, et al. Prospective study on the incidence, prevalence and 5-year pancreatic-related mortality of pancreatic cysts in a population-based study. Gut 2018;67:138-145. doi:10.1136/gutjnl-2016-313127

\section{PTU-77 UTILITY OF EUS-GUIDED THROUGH THE NEEDLE MICROBIOPSY IN INFLUENCING OUTCOMES OF PATIENTS WITH CYSTIC LESIONS}

Wei On*, Bharat Paranandi, Matthew T Huggett, Latifu Sanni, Alison Cairns. Leeds Teaching Hospitals NHS Trust, Leeds, UK

\subsection{6/gutjnl-2021-BSG.279}

Introduction The recent development of through-the-needle microbiopsy (TTNB) forceps via endoscopic ultrasound (EUS) has facilitated sampling of pancreatic cyst walls for histological analysis. This novel method has added to the armamentarium of the currently available radiological, biochemical and 


\begin{tabular}{lllllll}
\multicolumn{2}{l}{ Abstract PTU-77 Table 1} \\
\hline Patient & Location & Size $(\mathrm{mm})$ & Pre-TTNB MDT diagnosis & Post-TTNB histological diagnosis & Outcome & Adverse event \\
\hline 1 & HOP & 35 & IPMN vs. SCN & IPMN & Start surveillance & No \\
2 & TOP & 33 & Indeterminate & Indeterminate & Continue surveillance & No \\
$3^{*}$ & Retroperitoneal & 250 & GIST & GIST & Chemotherapy & Yes \\
4 & BOP & 35 & MCN & Lymphoepithelial cyst & Avoided surgery & No \\
5 & HOP & 40 & SB-IPMN vs. MCN & SCN & Avoided surveillance & No \\
6 & BOP & 50 & SB-IPMN & SCN & Avoided surveillance & No \\
7 & BOP & 40 & IPMN vs. MCN & MCN & Surgery & No \\
\hline
\end{tabular}

cytological modalities in the diagnostic algorithm of patients with pancreatic cystic lesions (PCL). We aimed to describe the utility of EUS-TTNB in influencing management outcomes at a tertiary hepatopancreatobiliary unit.

Methods A prospective database of consecutive patients who underwent EUS-TTNB from March 2020 to October 2020 was retrospectively analysed. Recorded variables included patient demographics, technical success, histological results, adverse events and management outcomes.

Results Seven patients (4 male; 3 female) were identified. All patients were discussed in dedicated multidisciplinary team (MDT) meetings and a consensus on the nature of the lesion was not possible. Seven patients had PCLs and one patient had a retroperitoneal cystic lesion. Technical success was achieved in $100 \%$ of patients. Specimen adequacy for definitive histological diagnosis was achieved in $85.7 \%$ of patients $(n=6)$, leading to a change in management. An adverse event was encountered in one patient* who developed an infection of the cystic lesion post EUS-TTNB. After extensive MDT discussion, EUS guided drainage was performed resulting in good clinical response. The table summarises the key characteristics and outcomes of the patients.

Conclusions Our case series has demonstrated EUS-TTNB to be a valuable and safe tool in the diagnostic pathway of patients with cystic lesions and led to a change in management in the majority of patients. Further larger prospective studies are required.

\section{Small bowel}

\section{PTH-1 SMALL-BOWEL NEUROENDOCRINE TUMOURS: THE ROLE OF DOUBLE-BALLOON-ENTEROSCOPY. A CASE SERIES FROM A TERTIARY REFERRAL CENTRE}

\begin{abstract}
${ }^{1,2}$ Rui de Sousa Magalhães, ${ }^{1}$ Alberto Murino, ${ }^{1}$ Alexandros Skamnelos, ${ }^{1}$ Rocio Chacchi Cahuin*, ${ }^{1}$ Andrea Telese, ${ }^{3}$ Edward Phillips, ${ }^{3}$ Faidon-Marios Laskaratos, ${ }^{4}$ TuVinh Luong, ${ }^{4} J e n n i f e r$ Watkins, ${ }^{1}$ Nikolaos Koukias, ${ }^{1}$ Regina Raymond, ${ }^{1}$ Nikolaos Lazaridis, ${ }^{3}$ Dalvinder Mandair, ${ }^{3}$ Christos Toumpanakis, ${ }^{3}$ Martyn E Caplin, ${ }^{1}$ Edward J Despott. 'Royal Free Unit for Endoscopy, The Royal Free Hospital and University College London (UCL) Institute for Liver and Digestive Health, London, UK; ${ }^{2}$ Gastroenterology Department, Hospital Senhora da Oliveira - Guimarães, Life and Health Sciences Research Institute (ICVS), School of Medicine, University of Minho, Braga, Portugal; ${ }^{3}$ Neuroendocrine Tumour Unit, ENETS Centre of Excellence, The Royal Free Hospital and University College London (UCL) Institute for Liver and Digestive Health, London, UK; ${ }^{4}$ Department of Cellular Pathology, The Royal Free Hospital and University College London (UCL) Institute for Liver and Digestive Health, London, UK
\end{abstract}

\subsection{6/gutjnl-2021-BSG.280}

Introduction The incidence of small bowel neuroendocrine tumours (SBNETs) is rising. In this context, double-balloon enteroscopy (DBE) appears to be critical for identification of the primary tumour, definitive histopathological diagnosis and lesion marking for subsequent surgical resection. Additionally enteroscopy provides a further assessment of the disease potential multifocality.

Methods Our aim was to evaluate the role of DBE in the management of SBNETs. Cohort-analysis was performed of consecutive patients with SBNETs who underwent DBE in our tertiary centre, from November 2012 to November 2019. Demographic, clinical, endoscopic findings and surgical data were analysed.

Results Eleven patients were included, 4 women; overall mean age of 56.2 years. Eight patients were referred for further investigation of mid gastrointestinal bleeding; 4 presented with overt bleeding and 4 presented with iron deficiency anaemia. Two cases were referred for further investigation of a lesion incidentally found on cross-sectional imaging. The final case was referred electively for localization of a primary tumour lesion, following previous resection of NET mesenteric metastasis. Twelve DBE procedures were performed (retrograde, $\mathrm{n}=9$; anterograde, $\mathrm{n}=3$ ). All DBEs successfully identified the primary lesion as per indication and submucosal tattoo was placed for surgical reference. All lesions were located within the ileum. Multifocal SBNETs identified with DBE were confirmed at surgical resection $(n=3)$. Histopathology samples taken through DBE were pathognomonic in all cases. The tattoo placed during DBE was identified in all surgical procedures thus facilitating 4 minimally-invasive tattoo-guided laparoscopic SB resections (mean resection length: $33.7 \mathrm{~cm}$ (SD $\pm 14.4 \mathrm{~cm}$ ) clearing 16 SBNETs.

Conclusions DBE has an important role to play within the diagnostic and management algorithm of SBNETs. Consequently enteroscopy could facilitate identification of multiple lesions and guide minimally invasive bowel-preserving surgical resection.

\section{PTH-2 BILE ACID MALABSORPTION AND SMALL INTESTINAL BACTERIAL OVERGROWTH IN PATIENTS WITH DIARRHOEA FOLLOWING PELVIC RADIOTHERAPY}

Andrea $\mathrm{Au}^{*}$, Caroline Henson. Manchester University NHS Foundation Trust, Manchester, UK

\subsection{6/gutjnl-2021-BSG.281}

Introduction Bile acid malabsorption (BAM) and small intestinal bacterial overgrowth (SIBO) are common causes of diarrhoea following pelvic radiotherapy ${ }^{1}$. BSG guidelines recommend 23-seleno-25-homotaurocholic acid (SeHCAT) and hydrogen breath testing for BAM and SIBO respectively as initial investigations in this patient group ${ }^{1}$. These tests seem to 